\title{
A Fuzzy Erlang Distribution Model for Parathyroid Hormone
}

\author{
A. Venkatesh ${ }^{1}$, S. Prakasam ${ }^{2}$ \\ Assistant Professors, Department of Mathematics, \\ A. V. V. M. Sri Pushpam College, Poondi, Thanjavur (Dt), \\ Tamilnadu.
}

\begin{abstract}
A mathematical modeling of biological system is essential to professionally increasing treatments for medical situation that parathyroid hormone .In this paper present a Erlang distribution approach to estimate the parameter and Erlang distribution based on fuzzy data that used in the formula for mean and variance of Erlang distribution was developed and it is used to find the effect of parathyroid hormone glands.
\end{abstract}

Key Words:- Erlang distribution, Parathyroid hormone(PTH), Mean, Variance.

\section{INTRODUCTION:}

The mathematical modeling in Biological systems is comparatively fresh expansion field. The 20th century has been a epoch of theatrical technological and scientific breakthroughs and discoveries. A common sentiment among scientists in all areas is that these developments will carry on, possibly most especially in the life and computer sciences.

The Erlang distribution was developed by to A.K. Erlang examine the number of telephone calls which might be made at the same time to the operators of the switching stations [5]. This vocation on phone traffic engineering has been lingering to consider waiting times in queuing systems in general. The distribution is as well used in the meadow of stochastic processes. The source of queuing hypothesis was in 1909, when A.K. Erlang (1878-1929) published his fundamental paper relating to the study of congestion in telephone traffic [1]. The journalism on the premise of queues and on the varied field of its applications has grown extremely over the years. The analysis for such an Erlangian queue is now folklore in the queuing literature. The Erlang distribution is the distribution of sum of exponential varieties. This distribution can be articulated as to come time and communication duration in telephone traffic. If the duration of individual calls are exponentially distributed then the duration of succession of calls is the Erlang distribution. The Erlang variate become Gamma variate when its shape limit is an integer .

The study [4] of the length of human life of Parathyroid gland, immunity etc, is a major importance in biological sciences. A substantial part of such study is devoted to the mathematical distribution of length of life by failure distribution. One of the problems of the present century which have serious implications for the next one, appears to be the PTH, which is affect of health and decreases throughout human life. Parathyroid glands are little endocrine glands in the collar of humans and other tetra pods. Humans frequently have four parathyroid glands, situated on the back of the thyroid gland in changeable location. The parathyroid gland produces and secretes parathyroid_hormone in reaction to a short blood calcium, which plays a key role in regulating the amount of calcium in the blood and within the bones. The concentration of extracellular ionized calcium is maintained within a narrow physiologic range by an exquisite system of negative and positive feedback regulators involving the major organs that transport calcium and phosphate, that is, the intestine, the kidneys, bone, and the endocrine organs, most prominently the parathyroid gland (PTG).

\section{ERLANG DISTRIBUTION:}

Erlang Distribution the shorthand $\mathrm{X} \sim$ Erlang $(\alpha, \mathrm{k})$ is second-hand to specify that the random variable $\mathrm{X}$ has the Erlang distribution with scale parameter $\alpha$ and shape parameter $\mathrm{n}$ [2]. An Erlang random variable X with scale parameter $\alpha$ and $\mathrm{k}$ stages has probability density function a random variable $\mathrm{X}$ has an Erlang-k $(\mathrm{k}=1,2, \ldots)$ distribution with mean $\mathrm{k} / \mu$. If $\mathrm{X}$ is the sum of $\mathrm{k}$ independent random variables $X_{1}, X_{2}, \ldots \ldots X_{K}$ having a common exponential distribution. The parameter $\mu$ is called the scale parameter, $\mathrm{k}$ is the shape parameter.

Poisson density function (PDF) of the erlang distribution is

$f_{x}(x)=\left\{\begin{array}{cc}\lambda^{k} x^{k-1} e^{-\lambda x} ; & x \geq 0 \\ 0 & \text { else }\end{array}\right.$
The mean of erlang distribution is $\mathrm{E}(\mathrm{X})=\frac{K}{\lambda}$ and The variance of erlang distribution is $\mathrm{V}[\mathrm{X}]=\frac{k}{\lambda^{2}}$. 
The $\alpha$ - cut of fuzzy mean for erlang distribution is

$$
\begin{aligned}
\bar{E}(\mathrm{X}) & =\left\{\bar{E}_{l}(\mathrm{X}), \bar{E}_{u}(\mathrm{x})\right\} \\
\text { where } \quad \bar{E}_{l}(\mathrm{X}) & =\min \left\{\begin{array}{c}
\bar{k} \\
\overline{\bar{\lambda}}
\end{array}\right\} \text { and } \bar{E}_{u}(\mathrm{X})=\max \left\{\begin{array}{c}
\bar{k} \\
\overline{\bar{\lambda}}
\end{array}\right\}
\end{aligned}
$$

The $\alpha$ - cut of fuzzy variance for the Erlang distribution is

$$
\begin{aligned}
\bar{V}(\mathrm{X}) & =\left\{\bar{V}_{l}(\mathrm{X}), \bar{V}_{u}(\mathrm{x})\right\} \\
\text { Where } \quad \bar{V}_{l}(\mathrm{X}) & =\min \left\{\frac{\bar{k}}{\overline{\lambda^{2}}}\right\} \text {, and } \bar{V}_{u}(\mathrm{X})=\max \left\{\frac{\bar{k}}{\overline{\lambda^{2}}}\right\}
\end{aligned}
$$

\section{APPLICATION:}

Let us consider an Experimental data reported in Estepa et al. [3], (adjusted and reproduced with permission of Kidney International). Ionized calcium concentrations were reduced by $0.4 \mathrm{mmol} / \mathrm{L}$ either in 30 min followed by a 120 -min hypocalcemic clamp. The fast $\mathrm{Ca} 2+$ reduction leads to a prominent peak in PTH levels. However, PTH levels during the hypocalcemic clamp following the fast reduction were significantly lower than PTH levels in the slow induction group.

The results were given in the following table.

Table 3.1 : PTH levels during the hypocalcemic clamp the fast reduction

\begin{tabular}{|l|l|l|l|l|l|l|l|l|l|}
\hline $\begin{array}{l}\text { Time } \\
\text { (Minutes) }\end{array}$ & 0 & 10 & 20 & 30 & 40 & 60 & 80 & 100 & 120 \\
\hline $\begin{array}{l}\text { PTH } \\
(\mathrm{Mg} / \mathrm{ml})\end{array}$ & 30 & 160 & 230 & 250 & 210 & 180 & 150 & 160 & 180 \\
\hline
\end{tabular}

\section{RESULTS:}

Consider the scale and shape parameter of erlang distribution for Table.3.1. are $\quad \mathrm{K}=6$ and $\lambda=26.723$

Let the corresponding fuzzy triangular numbers are

$$
\begin{aligned}
& \bar{K}=[5.7725,6,6.7836] ; \quad \bar{\lambda}=[26.2652,26.723,27.2667] \quad \text { The corresponding } \boldsymbol{\alpha} \text { (alpha)-cuts are } \\
& \bar{K}=[5.7725+0.2275 \alpha, 6.7836-0.7836 \alpha] \\
& \bar{\lambda}=[26.2652+0.4578 \alpha, 27.2667-0.5437 \alpha]
\end{aligned}
$$

The fuzzy mean for the PTH values are calculated from

$$
\bar{E}(\mathrm{X})=\left\{\bar{E}_{l}(\mathrm{X}), \bar{E}_{u}(\mathrm{x})\right\} \quad \text { and }
$$

Also the fuzzy variances are calculated from

$$
\bar{V}(\mathrm{X})=\left\{\bar{V}_{l}(\mathrm{X}), \bar{V}_{u}(\mathrm{x})\right\}
$$

Table 4.2: lower and upper fuzzy Mean of PTH

\begin{tabular}{|c|c|c|c|c|c|c|}
\hline$\alpha$ & low k & up k & low $\lambda$ & up $\lambda$ & low $\bar{E}(\mathrm{X})$ & up $\bar{E}(\mathrm{X})$ \\
\hline 0 & 5.7725 & 6.7836 & 26.2652 & 27.2667 & 0.219778 & 0.248787 \\
\hline 0.1 & 5.79525 & 6.70524 & 26.31098 & 27.21233 & 0.22026 & 0.246404 \\
\hline 0.2 & 5.818 & 6.62688 & 26.35676 & 27.15796 & 0.22074 & 0.244012 \\
\hline 0.3 & 5.84075 & 6.54852 & 26.40254 & 27.10359 & 0.221219 & 0.241611 \\
\hline 0.4 & 5.8635 & 6.47016 & 26.44832 & 27.04922 & 0.221697 & 0.239199 \\
\hline 0.5 & 5.88625 & 6.3918 & 26.4941 & 26.99485 & 0.222172 & 0.236778 \\
\hline 0.6 & 5.909 & 6.31344 & 26.53988 & 26.94048 & 0.222646 & 0.234348 \\
\hline 0.7 & 5.93175 & 6.23508 & 26.58566 & 26.88611 & 0.223118 & 0.231907 \\
\hline 0.8 & 5.9545 & 6.15672 & 26.63144 & 26.83174 & 0.223589 & 0.229457 \\
\hline 0.9 & 5.97725 & 6.07836 & 26.67722 & 26.77737 & 0.224058 & 0.226996 \\
\hline 1 & 6 & 6 & 26.723 & 26.723 & 0.224526 & 0.224526 \\
\hline
\end{tabular}




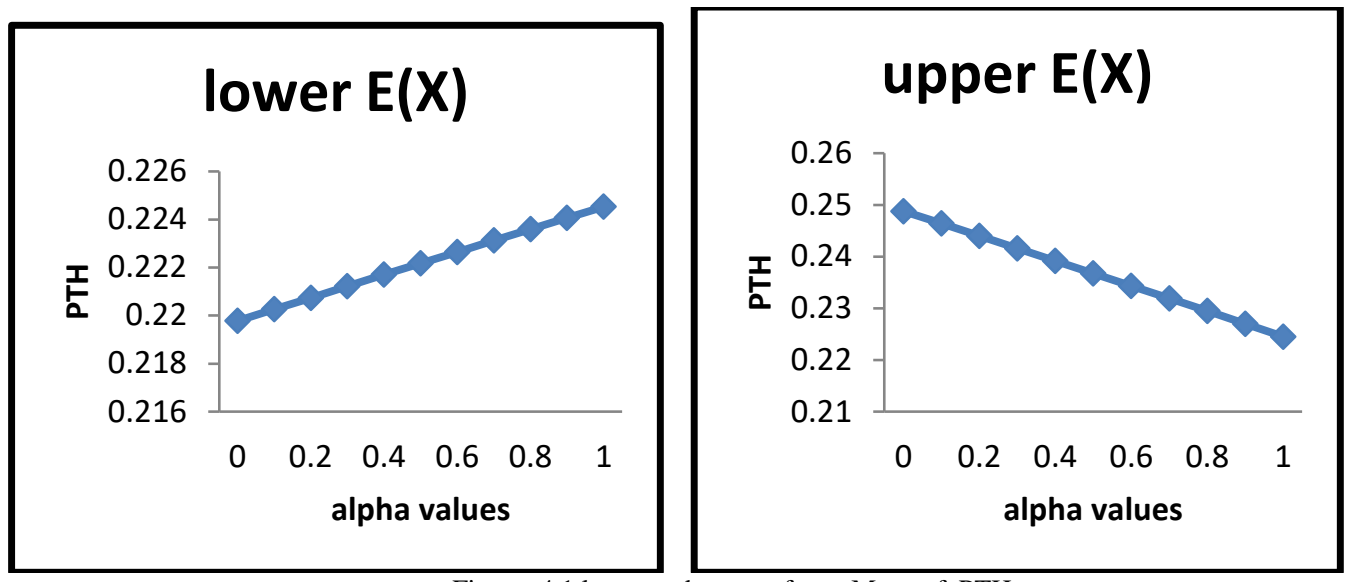

Figure :4.1 lower and upper fuzzy Mean of PTH

Table 4.3: lower and upper fuzzy variance of PTH

\begin{tabular}{|c|c|c|c|c|c|c|}
\hline$\alpha$ & low k & up k & low $\lambda$ & up $\lambda$ & low $\bar{V}(\mathrm{X})$ & up $\bar{V}(\mathrm{X})$ \\
\hline 0 & 5.7725 & 6.7836 & 26.2652 & 27.2667 & 0.008368 & 0.009124 \\
\hline 0.1 & 5.79525 & 6.70524 & 26.31098 & 27.21233 & 0.008371 \\
\hline 0.2 & 5.818 & 6.62688 & 26.35676 & 27.15796 & 0.008375 & 0.009055 \\
\hline 0.3 & 5.84075 & 6.54852 & 26.40254 & 27.10359 & 0.008379 & 0.008985 \\
\hline 0.4 & 5.8635 & 6.47016 & 26.44832 & 27.04922 & 0.008382 & 0.008843 \\
\hline 0.5 & 5.88625 & 6.3918 & 26.4941 & 26.99485 & 0.008386 & 0.008771 \\
\hline 0.6 & 5.909 & 6.31344 & 26.53988 & 26.94048 & 0.008389 & 0.008699 \\
\hline 0.7 & 5.93175 & 6.23508 & 26.58566 & 26.88611 & 0.008392 & 0.008626 \\
\hline 0.8 & 5.9545 & 6.15672 & 26.63144 & 26.83174 & 0.008396 & 0.008552 \\
\hline 0.9 & 5.97725 & 6.07836 & 26.67722 & 26.77737 & 0.008399 & 0.008477 \\
\hline 1 & 6 & 6 & 26.723 & 26.723 & 0.008402 & 0.008402 \\
\hline
\end{tabular}

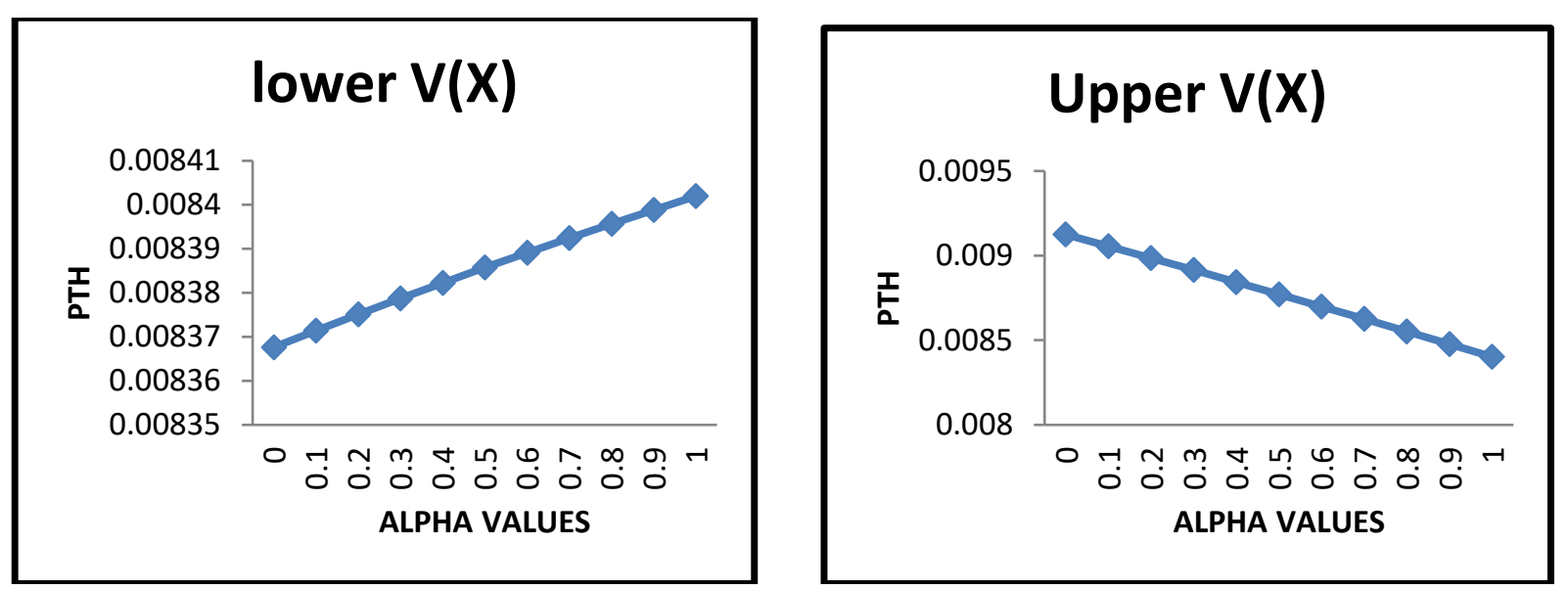

Figure :4.2 lower and upper fuzzy variance of PTH

\section{CONCLUSION :}

Here we successfully established the fuzzy models to calculate the effect of parathyroid hormone glands by the estimate of Mean and Variance with two parameter Erlang distribution. The results shows that the mean values are increased in lower $\alpha$ - cuts and decreased in upper $\alpha$ - cuts and the variance are increased in lower $\alpha$ - cuts and decreased in upper $\alpha$ - cuts for effect of parathyroid hormone on parathyroid gland. We validated our model by comparing its predictions with published experimental data under conditions during acute hypocalcemia, The lower mean and variance are increased but upper mean and variance are decreased where PTH Levels. 


\section{REFFERENCES:}

[1] Brockmeyer, E., Halstrom, H. L. And Jensen, A. , "The Life and Works of A. K. Earlang," 1948.

[2] Chrisian Walck, "Handbook on Statistical Distributions for Experimentalists", pp 69-70, 1996.

[3] Estepa, J.E. Aguilera - Tejero, Y.Almaden, M.Rodriguez, A.Felsenfeld, “ Effect of rate of Calcium reduction and hypocalcemic clamp on PTH secretion : a study in dog', Kidney Ins.55: pp 1724-1733, 1999.

[4] Gudrun Schappacher-Tilp, Alhaji Cherif, Doris H. Fuertinger, David Bushinsky \& Peter Kotanko, "A mathematical model of parathyroid gland biology', Physiological Reports, Volume 7, Issue 7, 2019.

[5] Oliver C.lbe, "Fundamentals of applied probability and random processes", pp 103- 158, 2014 